Leserbriefe

Onkologe 2021 $\cdot 27: 384$

https://doi.org/10.1007/s00761-021-00906-x

Angenommen: 20. Januar 2021

(c) Springer Medizin Verlag $\mathrm{GmbH}$, ein Teil von Springer Nature 2021

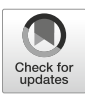

\section{Erwiderung}

Zum Leserbrief von G. Ullrich (2021) In Wahrheit bloß bedingter Respekt? Onkologe. https: //doi.org/10.1007/s00761-021-00905-y

\section{Originalpublikation}

Rabe A, van Oorschot B, Jentschke E (2020) Suizidalität bei Krebspatienten. Onkologe 26: 163-168. https://doi.org/10.1007/s00761020-00712-x

Wir bedanken uns für den Leserbrief von Herrn Dr. Ullrich zu unserem Artikel „Suizidalität bei Krebspatienten“. Die Zuschrift greift auch für uns sehr wichtige Punkte in dieser Thematik nochmals gezielt auf und bietet hilfreiche Ergänzungen. Gerne möchten wir auf die genannten Anmerkungen inhaltlich näher eingehen.

Bezüglich der Einschätzung einer „Überbetonung der Suizidalität bei Krebspatienten" begrüßen wir die in dem Leserbrief zitierten aufschlussreichen Ergebnisse der Studie von Henson et al. [1]. Auch wenn der vollendete Suizid bei onkologischen Patienten gemäß Henson et al. in relativ geringer Häufigkeit auftritt, ist diese deutlich erhöht gegenüber der nichtkrebserkrankten Gruppe, was aus unserer Sicht sowohl bemerkens- als auch erwähnenswert ist. Nicht zuletzt, da wir die Auffassung von Henson et al. teilen, wonach das erhöhte Suizidrisiko bei Patienten mit bestimmten Krebsarten ein erhebliches Problem potenziell vermeidbarer Todesfälle darstellt.

Auch schätzen wir die in der Zuschrift hervorgehobene Fragestellung, ob die aus der Psychiatrie gewonnenen Erkenntnisse und Einschätzungen ausreichend gül-

\title{
Elisabeth Jentschke
}

Comprehensive Cancer Center Mainfranken mit Interdisziplinärem Zentrum Palliativmedizin, Universitätsklinikum Würzburg, Würzburg, Deutschland

\section{Wenn das Leben nicht mehr als lebenswert empfunden wird}

tig auf die Situation Krebskranker übertragbar sind, als ein interessantes Forschungsgebiet ein, welchem es auch in Zukunft weiter nachzugehen gilt. Für die Befindlichkeit des Patienten sowie eine eventuelle (psychologische) Intervention ist allein die Gefühls- und Gedankenwelt des Patienten entscheidend - unabhängig davon, ob die Befindlichkeit des Patienten von Außenstehenden nachvollziehbar ist oder nicht.

Des Weiteren ist der Aussage „Ob ihm oder ihr die Möglichkeit einer palliativen Sedierung als bessere Option erscheint, bleibt die Entscheidung des Patienten, nicht unsere" aus unserer Sicht zuzustimmen. Nachdem die individuelle Situation des Patienten in einfühlsamer wie auch differenzierter Weise gemeinsam untersucht worden ist, eine umfassende medizinische und psychotherapeutische Behandlung behandelbarer Symptome vorgenommen worden ist sowie alle möglichen Handlungsoptionen eruiert worden sind, ist die vom Patienten getroffene Wahl in jedem Fall zu respektieren. Diesbezüglich möchten wir nochmals auf die von uns geschilderten Möglichkeiten zur Erhaltung der Würde des Patienten hinweisen: „Im Kontext nicht oder unzureichend behandelbarer Symptome besteht die Möglichkeit einer palliativen Sedierung. Hier ist jedoch nicht die Beschleunigung des Todes primäres Ziel, sondern die Linderung des Leidens und damit auch die Erhaltung der Würde“ und „Die würdezentrierte Therapie kann nachweislich das Würdeempfinden erhöhen ..." (S. 167).

Die im Leserbrief geschilderten Ergänzungen zur Suizidalität bei Krebspatienten befinden sich im Einklang mit unserer Haltung und unseren Ausführun- gen im Artikel. So ist aus unserer Sicht nochmals zu betonen: „Wenn das Leben nicht mehr als lebenswert empfunden wird, sollte man den Wunsch zu sterben respektieren und Maßnahmen wie das Beenden der Therapie oder der lebenserhaltenden Maßnahmen mit dem Patienten besprechen“ (S. 166).

\section{Korrespondenzadresse}

Dr. phil. Elisabeth Jentschke

Comprehensive Cancer Center Mainfranken mit Interdisziplinärem Zentrum Palliativmedizin,

Universitätsklinikum Würzburg

Würzburg, Deutschland

jentschke_e@ukw.de

Interessenkonflikt. E. Jentschke gibt an, dass kein Interessenkonflikt besteht.

\section{Literatur}

1. Henson KE, Brock R, Charnock J, Wickramasinghe B, Will O, Pitman A (2018) Risk of suicide after cancer diagnosis in England. JAMA Psychiatry 76(1):51-60. https://doi.org/10.1001/ jamapsychiatry.2018.3181 